\title{
Compressed Sensing-Based Tag Identification Protocol for a Passive RFID System
}

\section{$\operatorname{AUTHOR}(S)$ :}

Kaneko, Megumi; Hu, Wenhao; Hayashi, Kazunori; Sakai, Hideaki

\section{CITATION:}

Kaneko, Megumi ... [et al]. Compressed Sensing-Based Tag Identification Protocol for a Passive RFID System. IEEE Communications Letters 2014, 18(11): 2023-2026

\section{ISSUE DATE:}

2014-11

URL:

http://hdl.handle.net/2433/192478

\section{RIGHT:}

(c) 2014 IEEE. Personal use of this material is permitted. Permission from IEEE must be obtained for all other users, including reprinting/republishing this material for advertising or promotional purposes, creating new collective works for resale or redistribution to servers or lists, or reuse of any copyrighted components of this work in other works.; $こ 0$ 論文は出版社版でありません。引用の際には出版社版をご確認ご利用ください。; This is not the published version, Please cite only the published version. 


\title{
Compressed Sensing-based Tag Identification Protocol for a Passive RFID System
}

\author{
Megumi Kaneko, Wenhao Hu, Kazunori Hayashi and Hideaki Sakai \\ Graduate School of Informatics, Kyoto University, \\ Yoshida Honmachi Sakyo-ku, Kyoto, 606-8501, Japan \\ Email: \{meg,hu.wenhao,kazunori,hsakai\}@i.kyoto-u.ac.jp
}

\begin{abstract}
We propose a novel tag identification protocol based on Compressed Sensing (CS) in a Radio Frequency Identification (RFID) system. Unlike the existing Buzz protocol based on CS, our protocol does not require arbitrary restriction of the initially huge ID search space size. Moreover, by also making use of CS techniques for reducing the ID search space, our protocol significantly decreases the amount of required bits for successful identification as well as computational complexity compared to state-of-the-art protocols with ideal parameters. ${ }^{1}$
\end{abstract}

\section{INTRODUCTION}

Radio Frequency Identification (RFID) is an automated wireless identification technology which is being used in various industrial applications such as inventory control and supply chain management [1]. The advantage of RFID over other systems such as barcodes is that it requires no lineof-sight communication and consumes only very low power. In particular, in the passive RFID system, each RFID tag, providing a unique ID to its attached object, is activated by the radio waves transmitted from the RFID reader and performs processing and communication tasks by using the received power. One of the major issues in the identification process is the problem of collisions, which occur whenever multiple tags transmit their IDs simultaneously on the same radio resource, thereby reducing the efficiency of the RFID system shown in Fig. 1. This problem is more challenging in the passive RFID system, since each tag has low capabilities due to its battery-less configuration. There are two major approaches of anti-collision protocols, ALOHA-based and Binary Treebased approaches, which have been employed in existing RFID standards [1]. Both approaches can mitigate the collision problem to some extent, but their efficiency is still degraded in a system with a large number of tags. Therefore, [2] proposed a method where the single-antenna reader is able to detect the information of one tag out of two collided tag signals, leading to significant inventory time reduction.

Recently, a tag identification protocol named Buzz, based on the theory of Compressed Sensing (CS), was proposed in [3]. CS is a groundbreaking mathematical tool that is being increasingly used in the domains of digital signal and image processing, enabling a vector with correlated entries,

\footnotetext{
${ }^{1}$ This work was supported by the Grants-in-Aid for Scientific Research no. $23760334,26820143,24560457$ and 24560490 from the Ministry of Education, Science, Sports, and Culture of Japan.
}

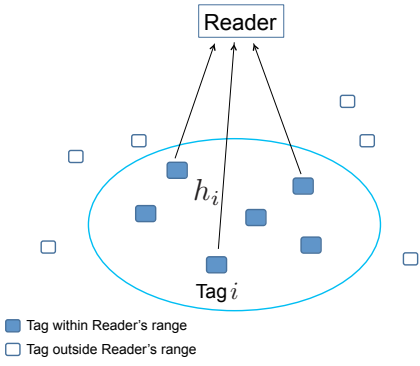

Fig. 1. RFID System

i.e., that can be transformed into a sparse vector through a transformation basis, to be recovered with high accuracy from a few random projections onto another, incoherent basis [4]. Unlike conventional anti-collision protocols, collisions are encouraged for exploiting CS-based techniques, by making the tags respond at the same time. In Buzz protocol, the initially huge ID search space (of size $2^{16}$ for a 16-bit ID) is firstly restricted based on the estimate of the number of tags to be identified $K$. Then, the candidate IDs are divided into groups, among which the ones containing tags to be identified are determined based on the tags' transmissions. Finally, the $K$ tag IDs are determined among the candidate tag IDs belonging to the remaining groups, using CS. One major drawback of Buzz is this initial requirement of search space restriction. Moreover, parameters are set either in an ad-hoc manner as in [3] or ideally with high computational complexity. Moreover, a large amount of time slots is required to determine the groups of tags to be identified, thereby reducing the overall efficiency.

In this work, we propose an improved protocol for tag identification based on CS, referred as the CS-ID protocol. The key point of this protocol is that it takes advantage of CS both for reducing the ID search space and for resolving the IDs in collision. It also operates without initial ID search space reduction, unlike Buzz. Simulation results show that the proposed protocol enables a significant reduction of the required amount of bits for successful identification, compared to ALOHA based protocols and Buzz.

\section{System MODEL}

We assume an RFID system as shown in Fig. 1 composed of $K$ tags within the reader's range and our goal is to identify these $K$ tags. The size of the temporary ID space is equal to $N=2^{16}$ as each RN16 ID is a 16 -bit sequence, according to the EPC Gen-2 standard [5]. Each tag picks a temporary 
ID among $N$. We define a binary vector $\mathrm{x}$ of size $N$ where $x_{i}=1$ if tag $i$ is one of the $K$ tags to be identified, $x_{i}=0$ otherwise. Thus, $\|\mathbf{x}\|_{0}=K$, i.e., there are $K$ nonzero elements in $\mathbf{x}$. The goal is thus to identify the elements $i$ for which $x_{i}=1$. After receiving a start command such as Query from the reader, each tag for which $x_{i}=1$ uses its ID as a seed in its hash function [1] to generate an $M \times 1$ pseudorandom binary vector $\mathbf{a}_{i}$ with elements $a_{i j} \in\{-1,+1\}, j=$ $1,2, \cdots, M$, where $j$ corresponds to the $j$ th element of $\mathbf{a}_{i}$ and $M$ denotes the number of bits required for successful identification. Gathering all vectors $\mathbf{a}_{i}$ in the transmission matrix $\mathbf{A}=\left[\begin{array}{llll}\mathbf{a}_{1} & \mathbf{a}_{2} & \ldots & \mathbf{a}_{N}\end{array}\right]$ of size $M \times N, M<N$, the received measurement vector $\mathbf{y}$ at the reader is given by

$$
\mathbf{y}=\mathbf{A H x}=\mathbf{A z},
$$

where $\mathbf{z}=\mathbf{H x}$, with $\mathbf{H}$ the diagonal channel matrix where $\mathbf{H}_{i i}=h_{i}$ denotes the complex channel coefficient for tag $i$, as each tag transmits in a narrowband channel $(\leq 640 \mathrm{kHz})$ [5].

CS tools enable to solve this underdetermined system of linear equations given the sparsity prior of the solution as $\|\mathbf{x}\|_{0}=K<<N$, by performing $\ell_{1}-\ell_{2}$ minimization [6],

$$
\left(P_{1-2}^{\lambda}\right): \quad \min _{\mathbf{z}} \lambda\|\mathbf{z}\|_{1}+\frac{1}{2}\|\mathbf{y}-\mathbf{A z}\|_{2}^{2},
$$

where $\|\mathbf{z}\|_{1}=\sum_{i=1}^{n}\left|z_{i}\right|$, and parameter $\lambda$ enables to make a trade-off between representation error and sparsity of the solution. Random matrices A whose entries are drawn from Gaussian or Sub-Gaussian (e.g., Bernoulli) distributions, guarantee stable recovery in the noisy case if the number of measurements $M$ obeys

$$
M \geq \alpha K \log (N / K),
$$

where $\alpha$ is a constant [4]. The Iterative ShrinkageThresholding Algorithm (ISTA) will be employed as it efficiently solves $\left(P_{1-2}^{\lambda}\right)$ with very low complexity [6]. However, note that this condition imposes a prohibitively large value of $M$ for $N=2^{16}$, the whole ID search space. This is why the reference and proposed protocols consider different ways of reducing the ID search space prior to applying CS techniques. Even then, the 16-bit RN16 IDs are not transmitted in the CS-based protocols, since in general, they will not fulfill the necessary condition (3) for successful recovery, which is why $M$-length pseudo-random sequences are generated and transmitted instead. One possible drawback of CS-based protocols is that these sequences need to be received synchronously at the reader. However, synchronization is not such a stringent requirement as low data rates are used for such applications. Finally, note that the considered hash function $h$ does not impose any restrictions on $M$, e.g., $M$ may be larger than 16. In addition, $h$ is assumed to guarantee that, if $I D \neq I D^{\prime}$, the probability that $h(I D) \neq h\left(I D^{\prime}\right)$ is extremely close to one, as confirmed in Section VI. In time slot $j$, each tag $i$ transmits the $j$ th bit $a_{i j}$.

\section{Reference Protocol: BuZZ}

Buzz protocol, a tag identification protocol based on CS techniques was proposed in [3]. As the knowledge of the

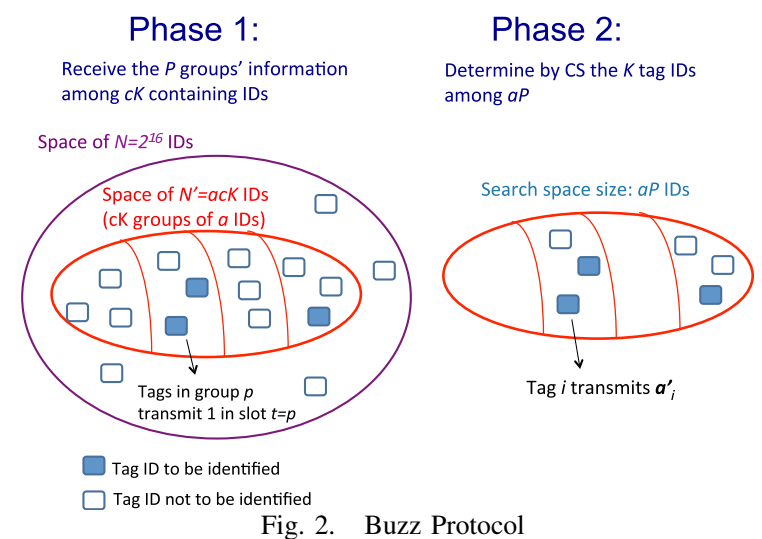

number of tags to be identified is required to restrict the initial ID search space, it performs the estimation of $K$ in the first step. Next, based on $K$, the size of the temporary ID search space is reduced from $N$ to $N^{\prime}=a c K$ where parameters $a$ and $c$ are chosen so that each tag gets a different ID with high probability. In [3] they were set to $a=K$ and $c=10$, without any clear justification. This is one of the major shortcomings overcome by the proposed protocol. Then, denoting by $\mathcal{I}$ the ID search space of size $a c K$, these IDs are divided into $c K$ groups using a hash function $h: \mathcal{I} \rightarrow\{1, . ., c K\}$, i.e., each ID is assigned a group number $p \in\{1, \ldots, c K\}$ where each group contains $a$ IDs, $h(I D)=p \in\{1, . ., c K\}$, as shown in Phase 1 of Fig. 2. Then, using $c K$ time slots, each time slot $t \in[1, c K]$ corresponding to a group, each tag transmits 1 in time slot $t=p$ if its ID is hashed in group $p$, and 0 otherwise. After reception of all $c K$ slots, the reader obtains the knowledge of the non-empty groups, i.e., the groups containing one or more IDs to be identified. There are at most $P \leq K$ non-empty groups, and since there are $a$ IDs per group, the size of the ID search space is reduced from $N^{\prime}$ to $a P$. Then, as in Phase 2 of Fig. 2, the IDs to be identified among $a P$ are determined based on CS techniques. Let $\mathbf{A}^{\prime}$ be the transmission matrix composed of the $a P$ possible pseudo-random ID sequences $\mathbf{a}^{\prime}{ }_{i}$ $(i=1,2, \cdots, a P)$, generated as in Section II, with elements $a_{i j}^{\prime} \in\{-1,+1\}, j=1,2, \cdots, M$. Then, the measurement vector $\mathbf{y}^{\prime}$ at the reader is given by (1), with $\mathbf{A}=\mathbf{A}^{\prime}$ and $\mathbf{z}=\mathbf{z}^{\prime}$ of size $a P$ is estimated as in (2).

From (3), CS theory guarantees successful reconstruction of $\mathbf{z}^{\prime}$ provided that $M \geq \beta K \log (a P / K)$, with a constant $\beta$.

\section{Proposed $C S$-ID PRotocol}

The proposed protocol is composed of two phases.

\section{Phase 1 of Fig. 3: CS-based Group Detection}

In order to detect the non-empty groups, $c K$ bits are required in Buzz protocol as each tag transmits one bit in the time slot corresponding to its group index $p$, while the maximum number of non-empty groups is $K$. By contrast, our protocol exploits the sparsity of the non-empty groups themselves, given that the number of non-empty groups, equal to $K$ at maximum, is sparse compared to the total number of groups $c K$, as $K<c K$. In this way, the required number of measurements for identification may be much reduced. Then, the protocol works as follows. 


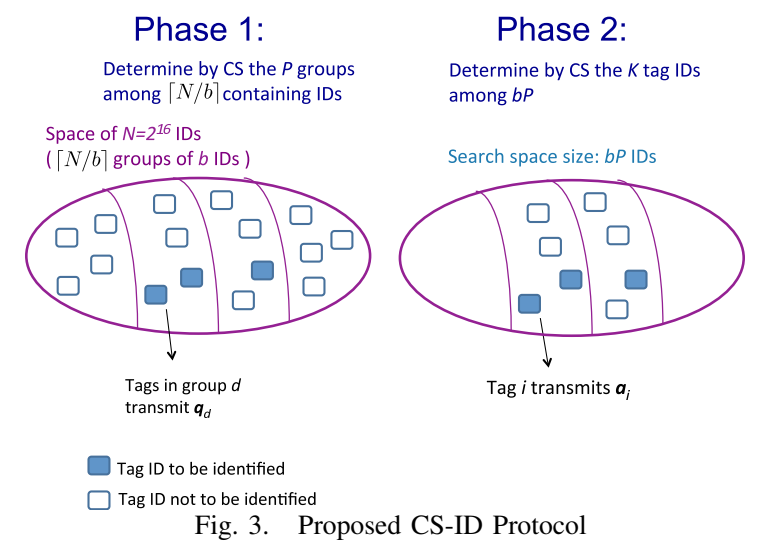

- Considering the whole temporary ID space $\mathcal{J}$ of size $N=$ $2^{16}$, all $N$ IDs are hashed into groups of size $b$ as

$$
h(I D)=d \in\{1, \ldots,\lceil N / b\rceil\} .
$$

In practice, this group division can be easily done by the Select command which selects a particular tag population based on tags' EPC or TID in User Memory [5].

- Using group index $d$, each tag uses it as a seed to generate a pseudo-random binary vector $\mathbf{q}_{d}$ with elements $q_{d j} \in$ $\{-1,+1\}, j=1,2, \cdots, M_{1}$ corresponding to the $j$ th time slot. The size of $\mathbf{q}_{d}$ is $M_{1}$, the number of time slots needed for successful group detection.

- After receiving the start signal, each tag transmits its group ID using one slot per bit, i.e., bit $q_{d j}$ in time slot $t=j$. The received signal at the reader can be written as

$$
\mathbf{y}_{1}=\mathbf{Q g},
$$

where $\mathbf{Q}$ of size $M_{1} \times\lceil N / b\rceil$, is the transmission matrix whose columns correspond to each group ID $\mathbf{q}_{d}, \mathbf{Q}=$ $\left[\begin{array}{llll}\mathbf{q}_{1} & \mathbf{q}_{2} & \ldots & \mathbf{q}_{\lceil N / b\rceil}\end{array}\right]$, and group vector $\mathbf{g}$ of size $\lceil N / b\rceil$, where

$$
g_{d}=\sum_{i=1}^{K} h_{i} e_{d i}, \text { where } e_{d i}= \begin{cases}1 & \text { if tag } i \in \text { group } d \\ 0 & \text { otherwise. }\end{cases}
$$

Note that the knowledge of channel gain $h_{i}$ is not required for CS recovery since $g_{d} \neq 0$ if at least one ID of a tag was hashed into group $d$, and $g_{d}=0$ otherwise.

- The reader uses $\mathbf{y}_{\mathbf{1}}$ to estimate $\hat{\mathbf{g}}$ as in (2).

We only need to receive about $M_{1}$ bits to decode $\mathbf{g}$, where $M_{1}$ should satisfy $M_{1} \geq \alpha_{1} K \log (N / b K)$ with constant $\alpha_{1}$. If there are $P$ non zero elements of $\hat{\mathrm{g}}$, i.e., $P$ non-empty groups, the corresponding $b P$ IDs remain as candidates.

\section{Phase 2 of Fig. 3: CS-based ID Decoding}

Thanks to the previous step, the ID search space was reduced from $N$ to $b P$ (but without initial reduction to $N^{\prime}$ as in Buzz). Next, each tag uses its own RN16 temporary ID as a seed to generate a pseudo-random binary vector which will serve as a new ID of length $M_{2}$ for enabling CS decoding. Similarly to the Group Detection step, we define transmission matrix $\mathbf{A}$ of size $M_{2} \times b P$ composed of IDs $\mathbf{a}_{i}$ with elements $a_{i j} \in$ $\{-1,+1\}, j=1,2, \cdots, M_{2}$, where each row corresponds to a time slot and each column corresponds to an ID. A is known at the reader since the space of all $M_{2}$-length IDs is known, as well as their partitioning into the fixed $\lceil N / b\rceil$ groups of $b$ $M_{2}$-length IDs, while the $P$ active groups are given by Phase 1 . We also define a vector $\mathbf{x}$ of size $b P$, corresponding to the possible $b P$ tags, where $x_{i}=1$ if tag $i$ is one of the $K$ tags to be identified, $x_{i}=0$ otherwise. Then, in Phase 2,

- Each tag to be identified sends its ID $\mathbf{a}_{i}$ of size $M_{2}$.

- The reader receives the signal

$$
\mathbf{y}_{2}=\mathbf{A z},
$$

from which the estimate $\hat{\mathbf{z}}$ is reconstructed as in (2).

We only need to receive about $M_{2}$ bits to decode $\mathbf{z}$, where $M_{2}$ should satisfy $M_{2} \geq \alpha_{2} K \log (b P / K)$, with constant $\alpha_{2}$.

\section{Discussion on the Number of Measurements}

In the proposed protocol, the total time slots usage which is equal to the number of bits or measurements, should satisfy

$M=M_{1}+M_{2} \geq \alpha_{1} K \log (N / b K)+\alpha_{2} K \log (b P / K)$.

The simulations give $\alpha_{1}, \alpha_{2} \approx 1$ for $99 \%$ reconstruction success. Hence, we can write $M \geq \alpha K \log \left(N P / K^{2}\right)$. As $P \leq K$ and $\alpha<2$, (8) is satisfied by $M_{\mathrm{CS}-\mathrm{ID}}=$ $2 K \log (N / K)$. On the other hand, the amount of time slots for Buzz should satisfy $M \geq c K+\beta K \log (a P / K)$, so we may set $M_{\mathrm{Buzz}}=c K+2 K \log (a)$ as $\beta<2$ from the simulations and $P \leq K$. Setting $a=K, c=10$ as in [3], we get $M_{\mathrm{Buzz}}=2 K \log \left(2^{5} K\right)$. Then, if the ID search space is set to $N=2^{16}$, we get $M_{\mathrm{CS}-\mathrm{ID}} \leq M_{\mathrm{Buzz}} \Longleftrightarrow 2^{11} \leq K^{2}$, which is satisfied for $K \geq 46$. Thus, theory shows that CSID may reduce the required overhead compared to Buzz. In practice, the next section will show that overhead reduction is achieved for $K \geq 15$. On the other hand, if the ID search space is reduced to $N^{\prime}=a c K=10 K^{2}$, the condition becomes $M_{\mathrm{CS}-\mathrm{ID}} \leq M_{\mathrm{Buzz}} \Longleftrightarrow 10 \leq 2^{5}$, which is always true.

\section{Numerical Results}

We compare the performance of the proposed CS-ID protocol with several reference protocols in terms of required number of bits $M$ for tag identification success rate over 0.99: Buzz protocol, Dynamic Frame Slotted ALOHA (DFSA) protocol, a tag identification protocol used in the EPC Gen-2 standard [5], and two enhanced versions of DFSA referred as DFSA-1 coll and DFSA-2coll. The pseudo-code for simulations is provided in [7]. AWGN channels with channel gains fixed to 1 were considered. In DFSA, tags are identified by the reader in several reading rounds. In each round, each tag randomly selects one time slot among $L$ in the frame and transmits its ID to the reader. In DFSA, only slots selected by exactly one tag enable identification. Thus, tags in collision slots have to retransmit again in the next rounds until they are successfully identified. The frame length $L$ is adapted at each round based on the estimated number of tags. In [8], it is shown that the optimal frame length $L_{\mathrm{opt}}$ at each round is equal to the number of tags $K_{r}$ remaining to be identified in round $r$ ( $K_{1}=K$ at round $r=1$ ). In DFSA-1coll based on [2], we further assume that the reader is able to identify one tag ID in two-tag collision slots, for which the optimal frame length 
is $L_{\mathrm{opt}, 1}=1+\sqrt{1+\frac{K_{r}\left(K_{r}-3\right)}{2}}$. Furthermore, DFSA-2coll enables detection of two tag IDs in two-tag collision slots, with optimal frame length $L_{\mathrm{opt}, 2}=\frac{3-K_{r}+\sqrt{\left(3-K_{r}\right)^{2}+4 K_{r}^{2}}}{2}$. Here, we will ideally assume the number of tags to be identified at each round $K_{r}$ to be known. Thus, Buzz protocol [3] will be evaluated without the $K$-estimation step, setting $a=K$ and the reduced temporary ID space of size $N^{\prime}=a c K$. Moreover, we evaluate the Buzz protocol with ideal a by optimizing the group size to $a_{\mathrm{opt}, K}=\min _{a}\left(\frac{N}{a}+\beta K \log a\right)$ by exhaustive search, i.e., at the expense of high computational complexity.

However, we will consider two cases for the proposed protocol: Case 1, without ID search space reduction, i.e., with search space size $N=2^{16}$, and Case 2, where the search space size is reduced to $N^{\prime}=a c K$, with $a=K, c=10$ as in [3]. Although not detailed here due to lack of space, another advantage of CS-ID protocol is that the total amount of required bits $M=M_{1}+M_{2}$ is independent of the group size $b$. Hence, we set $b=32$ without loss of generality. Then, for $N=2^{16}, K=30$ for example, we obtain $M_{1}=190$, $M_{2}=160$ for $99 \%$ recovery as explained in Section V.

\section{A. Case 1: full ID search space for CS-ID protocol}

Fig. 4 shows the required amount of bits for all protocols, with $N=2^{16}$ for $C S-I D$. The performance of DFSA is bad due to the higher occurrences of collisions as $K$ increases. $C S$ $I D$ also outperforms DFSA-1 coll which enables the detection of a tag ID in slots where two tags collide, and even DFSA2 coll where the two collided tag IDs can be detected. Compared to the performance of Buzz with $a=K$ and reduced temporary ID space of size $N^{\prime}=10 K^{2}, C S-I D$ requires a lower amount of bits for $K \geq 15$, as discussed in Section $\mathrm{V}$, while the gap widens as $K$ grows. Moreover, compared to the ideal performance of Buzz, $C S-I D$ also achieves a better performance, even though resolving $a_{\mathrm{opt}, K}$ for each $K$ in Buzz requires high computational complexity.

Note that for $M_{2}$-length sequences, the probability that none of the $K$ tags have the same sequence is equal to $\left(1-2^{-M_{2}}\right)^{K-1}$, which is extremely close to one even in CS-ID's worst case scenario where $M_{2}=30, K=5$. In particular, the probability that two tags get the same sequence is $2^{-M_{2}}=10^{-9}$, which is infinitely small in this context.

\section{B. Case 2: reduced ID search space for CS-ID protocol}

Fig. 5 shows that CS-ID protocol in Case 2 which uses the knowledge of $K$ for reducing the temporary ID space size to $N^{\prime}=10 K^{2}$, achieves the best performance. Thanks to this reduction, the overhead of $C S-I D$ is further decreased from Case 1, hence outperforming Buzz, ideal $a$, and a fortiori Buzz for all values for $K$, confirming the analysis of Section V.

Thus, the proposed protocol performs better than all reference algorithms in either cases. Moreover, $C S-I D$ with full ID search space performs even better than Buzz with reduced search space. With full search space, $C S-I D$ achieves a $23 \%$ reduction in average of the amount of required bits compared to $B u z z, a=K$, and a $40 \%$ reduction with reduced search space.

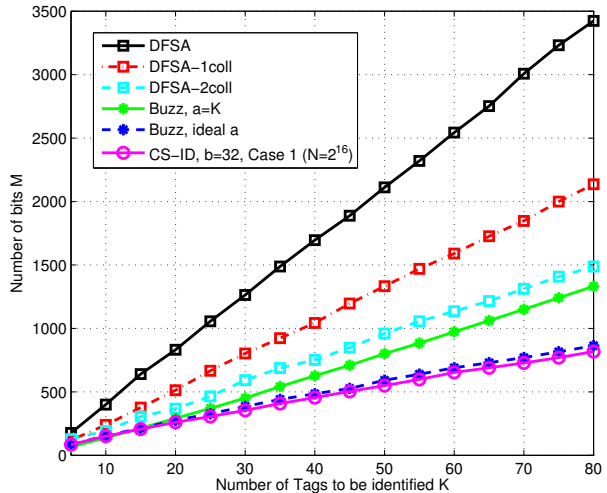

Fig. 4. Required amount of bits by the three protocols in Case 1

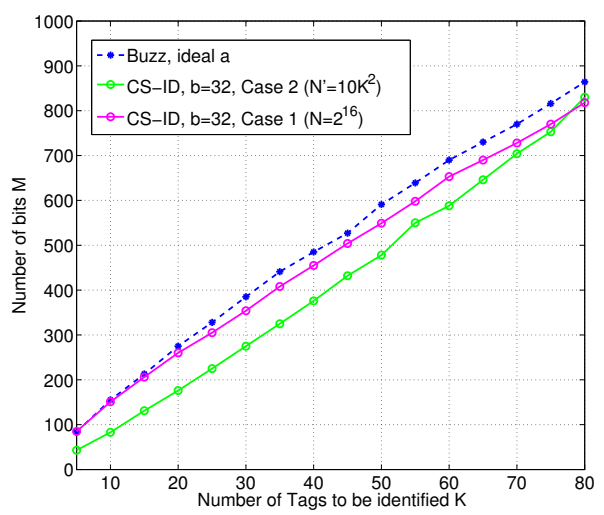

Fig. 5. Required amount of bits, comparison in Cases $1 \& 2$

\section{CONCLUSION}

We have proposed the $C S-I D$ protocol based on CS techniques for RFID tag identification. It improves the reference Buzz protocol by making use of CS for reducing the ID search space. Unlike Buzz, it is also able to perform identification without requiring any initial reduction of the huge ID search space size. Simulation results have shown the large performance gains offered by our protocol with much lower computational complexity compared to Aloha-based and Buzz protocols with ideal parameters.

\section{REFERENCES}

[1] Y. Zhang, L.T. Yang and J. Chen, RFID and Sensor Networks. CRC Press, Taylor \& Francis Group, 2010.

[2] A. Bletsas, J. Kimionis, A.G. Dimitriou and G.N. Karystinos, "SingleAntenna Coherent Detection of Collided FM0 RFID Signals," IEEE Trans. on Commun., vol. 60, no. 3, pp. 756 - 766, March 2012.

[3] J. Wang, H. Hassanieh, D. Katabi, P. Indyk, "Efficient and reliable lowpower backscatter networks," in ACM Sigcomm, Finland, August 2012.

[4] D.L. Donoho, "Compressed Sensing," IEEE Trans. on Info. Theory, vol. 52, no. 4, pp. 1289-1306, April 2006.

[5] EPCglobal Inc., EPC Radio-Frequency Identify Protocols Class-1 Generation-2 UHF RFID Protocol for Communications at $860 \mathrm{MHz}-$ 960MHz v1.1.0. Standard, 2005.

[6] M. Elad, Sparse and Redundant Representations. Springer, 2010.

[7] M. Kaneko, W. Hu, K. Hayashi and H. Sakai. (2014) CS-ID Algorithm Pseudo-Code. [Online]. Available: http://www.msys.sys.i.kyoto-u.ac.jp/ $\sim$ meg/CSID.pdf

[8] W.-T. Chen, "An accurate Tag Estimate Method for Improving the Performance of an RFID Anticollision Algorithm based on Dynamic Frame Length ALOHA," IEEE Trans. on Auto. Science and Eng., vol. 6, no. 1, pp. 9-15, January 2009. 